\title{
Innate Immune Response in Human Kidney Transplantation: IRF3 and IRF7 Together with Interferon-Alpha Are Significantly Up-Regulated in Acute Rejection
}

\author{
Paula Xavier Dias-Pintoํㅗ , José Gerardo G. Oliveira' ${ }^{2,3^{*}}$ \\ ${ }^{1}$ Instituto Português do Sangue e da Transplantação, Área Transplantação, Porto, Portugal \\ ${ }^{2}$ GCCT, Centro Hospitalar Universitário São João, Porto, Portugal \\ ${ }^{3}$ CINTESIS (Centro de Investigação em Tecnologias e Serviços de Saúde) FMUP (Faculdade de Medicina da Universidade do \\ Porto), Porto, Portugal \\ Email: *marilu@med.up.pt
}

How to cite this paper: Dias-Pinto, P.X. and Oliveira, J.G.G. (2021) Innate Immune Response in Human Kidney Transplantation: IRF3 and IRF7 Together with Interferon-Alpha Are Significantly Up-Regulated in Acute Rejection. Open Journal of Nephrology, 11, 379-396.

https://doi.org/10.4236/ojneph.2021.113031

Received: July 8, 2021

Accepted: August 24, 2021

Published: August 27, 2021

Copyright $\odot 2021$ by author(s) and Scientific Research Publishing Inc. This work is licensed under the Creative Commons Attribution International License (CC BY 4.0).

http://creativecommons.org/licenses/by/4.0/

(c) (i) Open Access

\begin{abstract}
Background: Ischemia-reperfusion injury of organ transplantation activates several mediators which may link the innate to the adaptive immune response. Down the cascade of TLRs, we selected to study the expression of Interferon Regulatory Factors (IRF)-3 and -7 inside human Kidney Transplanted (KTx) organs and the synthesis of IFN $\alpha$, the main growth factor induced by them, in KTx aspiration biopsy cultures. Simultaneously, we tested their robustness in diagnosing Acute Rejection (AR). Methods: Fine-needle aspiration biopsies (Fnab) were performed either on day 7 or 14 post-KTx among stable patients or on the day of AR diagnosis. On Fnab cytopreparations, we studied IRF3 and IRF7 by the enzymatic avidin-biotin complex staining, and in a different group of cases we quantified IFN $\alpha$ by ELISA in 48 hours Fnab culture supernatants. Results: AR group showed a significantly up-regulated expression for IRF3 and IRF7, reaching Positive Predictive Values (PPV) of 0.824 and 0.8, respectively, as well as Negative Predictive Values (NPV) above 0.9 for both; IFN $\alpha$ presented a PPV $=1.0$ and a NPV $=0.9$. A variation in the results was noticed according to different immunosuppressive therapies. Conclusions: Our findings suggest that IRF3 and IRF7, and IFN $\alpha$ which they promote, may be very important players in the early days post-KTx, linking the innate with an adaptive response and triggering acute rejection. These differences were very clear-cut, lending consistency to our speculation. It would be important to scrutinize for other potential effects derived from these IRFs up-regulation which could be of clinical relevance.
\end{abstract}




\section{Keywords}

Kidney Transplants, Aspiration Biopsies, Innate Immunity, IRF, IFN- $\alpha$

\section{Introduction}

A substantial proportion of the worldwide population may suffer from chronic kidney disease which was ranked among the first fifteen causes of death [1]. With few exceptions, kidney transplantation remain the best treatment alternative providing better long-term survival and better quality of life [2] [3], yet this procedure has been afflicted since its beginning by a serious mismatch between the demand and the offer.

Organ transplantation must overcome several barriers in order to achieve success, the most demanding being the immune response which must be modulated by immunosuppressive drugs and which can be divided into the innate and the adaptive response, that has received the lion part of our attention and has driven the most recent developments of currently used immunosuppressants in human organ transplantation. This being said, the first chronological challenge starts even when the organ has not yet been recovered and is enduring insults of diverse quality and gravity related to the donor dying process. This is to be aggravated by the cold ischemic period usually of several hours duration and sequentially by the reperfusion phase. This inevitable Ischemia and Reperfusion Injury (IRI) is highly determinant for non- or delayed function after transplantation [4] [5] and is associated with both T-cell mediated rejection as well as antibody-mediated rejection [6] [7].

IRI encompasses the innate immune response where Toll-Like Receptors (TLR) play an important role [8]. TLRs are transmembrane proteins and they belong to the interleukin-1 receptor superfamily. TLR2 and TLR4 are up-regulated in kidney tubular epithelial cells upon ischemia [9] [10] and TLR4 activation promotes the release and/or up-regulation of cytokines, chemokines, adhesion molecules and infiltration of neutrophils and macrophages [11] [12]. TLR activation promotes the downstream recruitment of various adapter molecules, namely the Toll-Interleukin 1 Receptor (TIR)-domain-containing adapter-inducing interferon$\beta$ (TRIF), inhibitor of the nuclear factor- $\mathrm{kB}$ kinase (IKK) and Tank-Binding Kinase-1 (TBK1). Together with other molecules acting upstream, TRIF, TBK1 and IKK $\varepsilon$ activate interferon regulatory factors IRF3 and IRF7 [13].

Sharing a great structural homology IRF3 and IRF7 are now acknowledged to be the principal mediators of interferon (IFN) induction. IRF3 is ubiquitously expressed, whereas IRF7 is expressed at very low levels with the exception of its abundant expression on plasmacytoid dendritic cells [14]. IRF3 can induce the expression of several cytokines in addition to IFN, namely interleukin (IL)-12, IL-15, IL-23 and CXCL10 whilst inhibiting TGF- $\beta$ [13] [15] [16], which may have important modulation in immune responses observed in organ transplan- 
tation, while IRF7 can either homodimerize or heterodimerize with IRF3 to induce IFN- $\alpha / \beta$ expression [17]. IRF3 and IRF7 have been found to play an important role in viral infection, inflammatory diseases and septic shock [18], whereas IRF7 seems to be needed for TLR9 activation and subsequent type I interferon synthesis in experimental SLE [17].

Recent evidence suggests that IRFs are stress sensors reacting to diverse pathophysiological stresses, including IRI [19]. During IRI, aberrantly localized DNA, such as that in the cytoplasm can activate IRF3 through a caspase-1 and stimulator of interferon genes dependent pathway with TBK1 [20]. IRF3 gene expression has been reported to be up-regulated during acute renal rejection [21], although it was reported to be non-significantly down-regulated together with IRF7 during acute rejection in liver transplantation [22].

As a result of IRF3 and IRF7 activation, INF $\alpha$ is produced, a cytokine endowed with pleiotropic effects beyond direct antiviral action, including the regulation of immune responses and modulation of the major histocompatibility complex I and II expression [23]. While IFN $\gamma$ has been the focus of important research by the transplant community IFN $\alpha$ has received much less attention. Blood circulating levels of IFN $\alpha$ were found to be lower among renal transplant patients [24]. Of greater interest, therapy with IFN $\alpha$ in transplant cases has been associated with the triggering acute rejection, more frequently with kidney transplants [25] than with liver transplants [26]. These events were observed several months post-transplantation, under stable clinical conditions and without changes in the immunosuppressive therapy [25] [26], a situation widely different comparing with the hectic early days of post-transplant surgery.

Previously, we reported that TLR-2 and TLR-9 expression was significantly down-regulated by the anti-IL-2 alpha chain-receptor antibody in Kidney Transplant patients (KTx) on day seven post-surgery and that by day 30 post-transplantation both TLR-4 and TLR-9 expression declined significantly [27] among rejectionfree cases. Also, we have reported that while no significant difference was observed for IL-12 by comparing rejection-free cases with acute rejection kidney transplants [28], a significant difference was found for IL-18, which was up-regulated during rejection [29]. Following our observations we decided to explore the behaviour of IRF3 and IRF7 and its main target, IFN $\alpha$ in fine-needle aspiration (Fnab) samples, IFN $\alpha$, which together with IL-18 could substitute for IL-12 in T lymphocyte priming.

\section{Patients and Methods}

This study included $151 \mathrm{KTx}, 87$ males and 64 females, between the ages of 20 and 68 years. Each patient provided adequate Fnab samples according to the criteria defined by P Haÿry [30] and all received an organ from a deceased donor. Table 1 summarizes patient's demographics and characteristics.

Each patient was treated from the outset with a calcineurin inhibitor, plus Mycophenolate Mofetil (MMF) and prednisolone, with the exception of the 
Table 1. Demographics and characteristics.

\begin{tabular}{|c|c|c|c|}
\hline Phenotype characteristic & $\begin{array}{l}\text { All Ktx recipients } \\
\qquad(\mathrm{n}=151)\end{array}$ & $\begin{array}{l}\text { Stable recipients } \\
\qquad(\mathrm{n}=106)\end{array}$ & $\begin{array}{l}\text { Rejection recipients } \\
\qquad(\mathrm{n}=45)\end{array}$ \\
\hline \multicolumn{4}{|l|}{ Gender } \\
\hline Female & 64 & 46 & 18 \\
\hline Male & 87 & 61 & 26 \\
\hline \multicolumn{4}{|l|}{ Cause of ESRD } \\
\hline $\mathrm{DN}$ & 29 & 22 & 7 \\
\hline $\operatorname{IgA}$ & 16 & 11 & 5 \\
\hline RPGN & 11 & 5 & 6 \\
\hline SLE & 5 & 3 & 2 \\
\hline FSGS & 8 & 7 & 1 \\
\hline TIN & 39 & 28 & 11 \\
\hline HTN & 6 & 4 & 2 \\
\hline CGN & 25 & 19 & 6 \\
\hline PKD & 12 & 9 & 3 \\
\hline \multicolumn{4}{|l|}{ Immunosupression } \\
\hline CsA & 107 & 78 & 29 \\
\hline RAPA & 15 & 14 & 1 \\
\hline TAC & 62 & 46 & 16 \\
\hline Anti-IL2 $\alpha \mathrm{R}$ & 23 & 21 & 2 \\
\hline \multicolumn{4}{|l|}{$\mathrm{Ktx}$} \\
\hline First & 139 & 102 & 37 \\
\hline Re-Ktx & 12 & 7 & 5 \\
\hline DGF & 13 & 11 & 2 \\
\hline Serum Creatinine $(\mathrm{mg} / \mathrm{dL})$ & NA & 3.3 & 5.1 \\
\hline
\end{tabular}

Values are reported in the given units and all comparisons between groups were non-significant, except serum creatinine that was higher in recipients with $\mathrm{AR}(\mathrm{P}=0.042)$. Recipients are all adult, aged between 20 68 years old and transplanted with kidney deceased donors. AR diagnosis were done on the biopty-gun biopsy, done at the same time as Fnab, classified according to Banff criteria and secured by a positive response to treatment or by histologic reassessment of graft nephrectomy. DN: diabetes mellitus, IgA: IgA nephropathy, RPGN: rapidly progressive glomerulonephritis, SLE: systemic lupus erythematous, FSGS: focal segmental glomerulosclerosis, TIN: tubulointerstitial nephritis, HTN: hypertension, CGN: chronic glomerulonephritis, PKD: adult polycystic kidney disease, DGF: delayed graft function.

second grafts that received quadruple sequential therapy, including two to five doses of thymoglobulin, according to the number of blood lymphocytes; also, in 15 first KTx Rapamycin (RAPA) substituted for MMF, and in twenty-three of first KTx an IL-2R $\alpha$-chain antibody was added. The therapeutic target whole blood levels for CsA, TAC and RAPA during the first three months post-KTx were $150-250 \eta \mathrm{g} / \mathrm{ml}, 6-12 \eta \mathrm{g} / \mathrm{ml}$ and $4-12 \eta \mathrm{g} / \mathrm{ml}$, respectively. Moreover, all patients had a panel of reactive antibodies less than $10 \%$ with the exception of second KTx. 
One hundred and twenty-four KTx remained rejection-free for the first year post-KTx, at least, 78 treated with CsA and 46 with TAC, including 14 cases treated with RAPA, and 21 with IL-2R $\alpha$-chain antibody. Forty-five KTx developed an acute rejection episode at a median of $13.5 \pm 415$ days post-KTx, 29 episodes occurred during the first month, six cases during the second and third month, and ten cases after the third month post-KTx. Twenty-nine of these acute rejection cases were treated with CsA and sixteen with TAC, including one patient treated with RAPA, two with IL-2R $\alpha$-chain antibody, and three with quadruple therapy. Every acute rejection episode was diagnosed by a biopty-gun biopsy done at the same time as the Fnab procedure and read by an independent pathologist following the standardized Banff criteria. The rejection diagnosis was further secured by either a positive response to treatment or by histologic reassessment of graft nephrectomy. Acute rejection was treated with either: 1) 3 pulses of IV 250 - $500 \mathrm{mg}$ methylprednisolone; 2) thymoglobulin, minimum of two doses; or 3) 5 - 12 sessions of plasmapheresis and IgG at $0.4 \mathrm{gr} / \mathrm{kg}$ weight, if the episode was graded IIa or greater or when c4d positivity was observed along with donor-specific antibodies. Only two cases proved to be treatment resistant, and both patients had their graft surgically removed before the first month post-KTx.

All patients received prophylaxis with ganciclovir/valganciclovir when the donor was positive and the recipient was negative for CMV, and whenever thymoglobulin was administered. Furthermore, each KTx received cotrimoxazol as prophylaxis for Pnemocystis jirovecii during the first six to twelve months postsurgery.

Among the rejection-free cases, Fnab were done on day seven (in close to $80 \%$ of cases) and on days 14 or 30 post-KTx for the remaining KTx, 90 - 150 min after the morning intake of immunosuppressive drugs, and on the day of the biopty-gun biopsy among rejection cases. The corresponding blood sample was drawn with the Fnab procedure. As a rule, one patient provided one sample for analysis.

\subsection{Immunocytochemistry Studies}

The Fnab samples was submitted to a $700 \mathrm{rpm}$ cytocentrifugation for $10 \mathrm{~min}$ and kept at $-70^{\circ} \mathrm{C}$ until testing.

The cytoslides were brought back to room temperature and they were submitted to the immunocytochemistry studies by the enzymatic Avidin Biotin Complex (ABC) method using the detection system UltraVision ${ }^{\mathrm{TM}}$, HRP/DAB (Horseradish Peroxidase/Three, 3'Diaminobenzidine Tetrahydrochloride) from Thermo Scientific, UK. All the incubations were done at room temperature. Briefly, the cytoslides were hydrated in ethanol $95^{\circ}$ and incubated with hydrogen peroxide for $15 \mathrm{~min}$ to peroxidase blocking, followed by a rinse in distilled water and Tris Buffered Saline (TBS) solution at $\mathrm{pH}=7.4$. Then, for blocking unspecific immunoglobulins, it was done $10 \mathrm{~min}$ incubation with Ultra $\mathrm{V}$ Block from Lab Vision ${ }^{\mathrm{TM}}$. After removal of the excess of the unspecific serum, the primary 
antibody at the appropriated dilution was added for $60 \mathrm{~min}$ incubation. In the end, the cytoslides were washed in distilled water and dipped for $10 \mathrm{~min}$ in TBS before 10 min of incubation with the secondary antibody, at a concentration of 4 $\mu \mathrm{g} / \mathrm{ml}$ of rabbit anti-goat. After rinse of the secondary antibody excess with TBS, a new incubation for $10 \mathrm{~min}$ with Streptavidin Peroxidase followed with $10 \mathrm{~min}$ rinse in TBS and then the addition of DAB Chromogen and DAB Substrate for 10 min incubation. Finally, hematoxylin from Mayer's Hematoxylin, Dako Cytomation was applied followed by a rinse in running tap water for $2 \mathrm{~min}$ and one min dehydration with ethanol $95^{\circ}$, and a coverslip with Entellan ${ }^{\circledR}$ mounting medium. The primary antibodies used included a goat polyclonal IgG at $40 \mu \mathrm{g} / \mathrm{ml}$, for IRF3 and a goat polyclonal IgG at $4 \mu \mathrm{g} / \mathrm{ml}$ for IRF7, both acquired from Santa Cruz Biotechnology. From each cytoslide every kidney tubular cell (R) was counted as well as every lymphocyte and monocyte-macrophage (L/M), both negative and positive for the antibody in order to present the absolute values of positive cells as well as the ratio of positive cells for both R cells and for L-M cells in an attempt to correct for the variation in cellular Fnab sample contents.

\subsection{ELISA Studies in Fnab Culture Supernatants}

Fnab samples were cultured as previously described (28). Briefly, the samples were aspirated into $6 \mathrm{ml}$ of RPMI medium with heparin lithium at $125 \mathrm{U} / \mathrm{ml}$ and after red blood cell lysis the crude cell suspensions were adjusted to a final concentration of $5 \times 10^{4}$ cells/200 $\mu$ l. (Typically, each Fnab had between 0.3 to $1.0^{6}$ cells $/ \mathrm{ml}$ and samples from acute rejection carried higher cell numbers than the others procured from stable cases). Fnab cultures were done in RPMI medium supplemented with penicillin, gentamicin and L-glutamine, 10\% of autologous serum obtained from the blood sample drawn concurrently with Fnab procedure and $10 \mathrm{U} / \mathrm{ml}$ of rIL-2. After 48 hours of incubation at $37^{\circ} \mathrm{C}$ and $5 \%$ of $\mathrm{CO}_{2}$ supernatants were collected and kept at $-70^{\circ}$ until testing.

The IFN $\alpha$ study in Fnab culture supernatants was done by ELISA according to the manufacturer's instruction, PBL Biomedical Laboratories, employing microtiter plate wells coated with purified human IFN $\alpha$ standard and using $100 \mu \mathrm{l}$ for each test sample.

\subsection{Statistical Analysis}

The statistical analysis included the determination of median, SD, and interquartile ranges. The comparisons for serum creatinine and whole blood immunesuppressor drugs levels were done by unpaired Student's T test, and the comparative analysis for IRF3, IRF7, and IFN $\alpha$ results by Mann-Whitney U test. The correlations between the IRFs and IFN $\alpha$ with serum creatinine and blood drug levels were tested using Spearman correlation. The sensitivity, specificity, Negative Predictive Value (NPV) and Positive Predictive (PPV) and areas under the ROC were evaluated when indicated.

This study was approved by the Faculty of Medicine of University of Porto 
and University Hospital of S. João, Porto joint Committee of Ethics. Informed consent was obtained from each patient and the study was conducted in compliance with the Good Clinical Practice Guidelines declaration of Helsinki and Istanbul.

\section{Results}

The median and interquartile range for serum creatinine among rejection-free cases on day seven post-KTx was $3.8 \mathrm{mg} / \mathrm{dl}$ and $1.4-7.4 \mathrm{mg} / \mathrm{dl}$, respectively and $5.1 \mathrm{mg} / \mathrm{dl}$ and $2.4-10 \mathrm{mg} / \mathrm{dl}$, respectively for acute rejection group $(\mathrm{P}=0.01)$; $2.7 \mathrm{mg} / \mathrm{dl}$ and $1.4-10 \mathrm{mg} / \mathrm{dl}$ on days 14 and 30 post-KTx combined $(\mathrm{P}>0.05$ on comparing stable cases on day seven with day $14-30$ combined, $\mathrm{P}=0.01$ when comparing day 14 - 30 combined with acute rejection day). Nineteen cases of delayed graft function were remarked among rejection-free cases and eleven among acute rejection cases. The whole blood levels for CsA, TAC and RAPA were within the limits of the transplant unit protocol in almost every case and no episode of calcineurin toxicity or of clinical important CMV disease occurred. However, both CsA and TAC blood levels were significantly inferior among acute rejection as compared with all the rejection-free KTX, P = 0.001 and $\mathrm{P}=$ 0.004 , respectively. No significant difference was observed when comparing HLA matching for rejection-free cases with acute rejection cases but a significant correlation was observed between the presence of anti-HLA antibodies pre-KTx and acute rejection $(\mathrm{P}=0.004)$.

In Table 2 we present the IRF3 results. A highly significant difference was observed concerning IRF3 expression which was up-regulated in acute rejection patients, whether relating the absolute numbers of positive cells, $\mathrm{P}=0.0002$, ratio of positive cells over R cells, $\mathrm{P}<0.0001$, or ratio of positive cells over LM cells, $\mathrm{P}<0.0001$. Despite a down-regulation of IRF3 expression was noticed among IL-2R $\alpha$-chain antibody treated cases, this did not reach a statistically significant level. Also, no correlation was observed between blood drug levels and IRF3 expression, as well as a positive but non-significant correlation was observed between IRF3 expression and creatinine values among acute rejection cases, $r=0.29$.

In Table 3 we present the findings concerning IRF7. A significant up-regulation of IRF7 was noticed among acute rejection cases. Yet, there was non-significant down-regulation of IRF7 among treated KTx as compared to triple therapy, but a significant difference was observed comparing the absolute numbers of positive cells counted among stable triple therapy and those with stable IL-2R $\alpha$-chain antibody treatment $(\mathrm{P}=0.02)$ and the ratio positive cells over lymphocyte/monocytes $(\mathrm{P}=0.023)$. This up-regulation of IRF7 expression was the opposite observed for IRF3 expression among IL-2R $\alpha$-chain antibody treated KTx. Even so, a highly diagnostic performance was found for the absolute values of IRF7 expression using a cut-off of 35 cells. Of difficult comprehension was the positive correlation, 0.56 , between the absolute number of positive cells for 
Table 2. Results for IRF3 expression in Fnab cells.

\begin{tabular}{|c|c|c|c|c|c|}
\hline & $\begin{array}{c}\text { A } \\
\mathrm{n}=36\end{array}$ & $\begin{array}{c}\mathrm{A} 1 \\
\mathrm{n}=21\end{array}$ & $\begin{array}{c}\text { A2 } \\
\mathrm{n}=15\end{array}$ & $\begin{array}{c}\text { B } \\
\mathrm{n}=17\end{array}$ & $\begin{array}{c}\text { Mann-Whitney } \\
\text { P }\end{array}$ \\
\hline Absolute number & $\begin{array}{c}51.9 \pm 38.5 \\
{[21-76]}\end{array}$ & $\begin{array}{l}60.6 \pm 40 \\
{[31-78]}\end{array}$ & $\begin{array}{c}36.7 \pm 27.3 \\
{[16-53]}\end{array}$ & $\begin{array}{c}156 \pm 74 \\
{[110-188]}\end{array}$ & $\begin{array}{l}A 1 \text { vs } A 2: 0.07 \\
A \text { vs } B: 0.0002\end{array}$ \\
\hline PosCells/Rcells & $\begin{array}{c}0.16 \pm 0.15 \\
{[0.04-0.16]}\end{array}$ & $\begin{array}{l}0.18 \pm 0.15 \\
{[0.07-0.3]}\end{array}$ & $\begin{array}{l}0.21 \pm 0.24 \\
{[0.04-0.3]}\end{array}$ & $\begin{array}{c}1.4 \pm 1.9 \\
{[0.4-1.14]}\end{array}$ & $\begin{array}{l}A 1 \text { vs } A 2: 0.42 \\
A \text { vs } B: 0.0001\end{array}$ \\
\hline PosCells/LM & $\begin{array}{c}0.18 \pm 0.2 \\
{[0.06-0.2]}\end{array}$ & $\begin{array}{c}0.22 \pm 0.24 \\
{[0.08-0.23]}\end{array}$ & $\begin{array}{c}0.17 \pm 0.17 \\
{[0.05-0.22]}\end{array}$ & $\begin{array}{c}0.64 \pm 0.34 \\
{[0.29-1]}\end{array}$ & $\begin{array}{l}A 1 \text { vs } A 2: 0.50 \\
A \text { vs } B: 0.0001\end{array}$ \\
\hline
\end{tabular}

Group A: all stable KTx; A1: Fnab on day 7, triple therapy; A2: Fnab with IL-2R $\alpha$-chain antibody therapy; group B: acute rejection group. Results expressed as absolute number of positive cells, ratio of positives over renal parenchymal cells (R), ratio of positives over total of lymphocyte-monocyte/macrophage cells (LM); values given as median $\pm \mathrm{SD}$ and lower and upper quartiles between brackets.

Table 3. Results for IRF7 expression in Fnab cells.

\begin{tabular}{|c|c|c|c|c|c|c|}
\hline & $\begin{array}{c}\text { A } \\
\mathrm{n}=21\end{array}$ & $\begin{array}{c}\text { A1 } \\
\mathrm{n}=8\end{array}$ & $\begin{array}{c}\text { A2 } \\
\mathrm{n}=7\end{array}$ & $\begin{array}{c}\text { A3 } \\
\mathrm{n}=6\end{array}$ & $\begin{array}{c}\text { B } \\
\mathrm{n}=13\end{array}$ & $\begin{array}{c}\text { Mann-Whitney } \\
\text { P }\end{array}$ \\
\hline Absolute number & $\begin{array}{c}17.2 \pm 33 \\
{[1-9]}\end{array}$ & $\begin{array}{l}4.8 \pm 4.3 \\
{[1.5-7]}\end{array}$ & $\begin{array}{c}2.8 \pm 3.1 \\
{[0-6]}\end{array}$ & $\begin{array}{l}54.6 \pm 44 \\
{[33-67]}\end{array}$ & $\begin{array}{l}83.4 \pm 72 \\
{[37-95]}\end{array}$ & $\begin{array}{c}A 1 \text { vs } A 3: 0.020 \\
A 2 \text { vs } A 3: 0.014 \\
A \text { vs } B: 0.0008\end{array}$ \\
\hline PosCells/Rcells & $\begin{array}{c}0.08 \pm 0.1 \\
{[0.01-0.1]}\end{array}$ & $\begin{array}{l}0.07 \pm 0.08 \\
{[0.01-0.1]}\end{array}$ & $\begin{array}{c}0.015 \pm 0.01 \\
{[0-0.03]}\end{array}$ & $\begin{array}{l}0.18 \pm 0.17 \\
{[0.1-0.21]}\end{array}$ & $\begin{array}{c}0.38 \pm 0.62 \\
{[0.14-0.35]}\end{array}$ & $\begin{array}{c}A 1 \text { vs } A 3: 0.19 \\
A 2 \text { vs } A 3: 0.009 \\
A \text { vs } B: 0.002\end{array}$ \\
\hline PosCells/LM & $\begin{array}{c}0.06 \pm 0.09 \\
{[0.01-0.04]}\end{array}$ & $\begin{array}{c}0.02 \pm 0.02 \\
{[0.01-0.03]}\end{array}$ & $\begin{array}{c}0.01 \pm 0.01 \\
{[0-0.02]}\end{array}$ & $\begin{array}{l}0.19 \pm 0.09 \\
{[0.2-0.26]}\end{array}$ & $\begin{array}{l}0.8 \pm 0.86 \\
{[0.14-1]}\end{array}$ & $\begin{array}{c}A 1 \text { vs } A 3: 0.023 \\
A 2 \text { vs } A 3: 0.009 \\
A \text { vs } B: 0.0002\end{array}$ \\
\hline
\end{tabular}

Group A: stable KTx; A1: Fnab on day 7, standard triple therapy; A2: Fnab, treated with quadruple (thymoglobulin) therapy; A3: treated with IL-2R $\alpha$-chain antibody; group B: acute rejection group. Results expressed as absolute number of positive cells, ratio of positives over (R) cells, ratio of positives over total of lymphocyte-monocyte/macrophage cells (LM); values given as median \pm SD and lower and upper quartiles between brackets.

IRF7 and calcineurin inhibitor drug levels among stable KTx and a negative correlation, -0.53 , when restricted to acute rejection $\mathrm{KTx}$. This negative correlation was also clearly observed for the ratio of positive cells over lymphocyte-monocytes and calcineurin inhibitor drug levels in acute rejection, $\mathrm{r}=-0.87$.

In Table 4 and Table 5 are the results for acute rejection diagnosis performance, following the analysis for cut-off points, for IRF3 and IRF7 respectively, which reached very high values. The best diagnostic performance was achieved by the ratio of positive cells for IRF3 over the renal parenchymal cells, the optimal cut-off being 0.382 translated into a positive predictive value of 0.824 and a negative predictive value of 0.912. Also, in Figure 1 and Figure 2, we show the results assessed for AUC of the ROC curves and distribution of non-rejection patients and acute rejection group, either for absolute numbers of positive cells, ratio of positive cells over R cells or ratio of positive cells over LM cells, for IRF3 and IRF7 respectively. 
Table 4. IRF3 diagnostic performance.

\begin{tabular}{cccc}
\hline IRF3 & Absolute number & Pos cells/Rcells & Pos cells/LM \\
\hline Sensitivity & 0.824 & 0.824 & $\mathbf{0 . 9 4 1}$ \\
Specificity & $\mathbf{0 . 8 8 2}$ & $\mathbf{0 . 9 1 2}$ & 0.735 \\
Positive predictive value & 0.778 & 0.824 & 0.640 \\
Negative predictive value & $\mathbf{0 . 9 0 9}$ & $\mathbf{0 . 9 1 2}$ & $\mathbf{0 . 9 6 2}$ \\
Positive likelihood ratio & 7.00 & 9.333 & 3.556 \\
Negative likelihood ratio & 0.200 & 0.194 & 0.080 \\
\hline
\end{tabular}

The cut-off points for absolute number of positives, ratio of positives over number of renal cells (R) and ratio of positive cells over number of lymphocytes plus monocytes-macrophages (LM) were 93, 0.382 and 0.187 , respectively.

Table 5. IRF7 diagnostic performance.

\begin{tabular}{cccc}
\hline IRF7 & Absolute number & Pos cells/Rcells & Pos cells/LM \\
\hline Sensitivity & $\mathbf{0 . 9 2 3}$ & 0.769 & 0.692 \\
Specificity & $\mathbf{0 . 8 5 0}$ & 0.800 & 1.000 \\
Positive predictive value & 0.800 & 0.714 & $\mathbf{1 . 0 0 0}$ \\
Negative predictive value & $\mathbf{0 . 9 4 4}$ & 0.842 & 0.833 \\
Positive likelihood ratio & 6.15 & 3.846 & $\infty$ \\
Negative likelihood ratio & 0.090 & 0.288 & 0.308
\end{tabular}

The cut-off points for absolute number of positives, ratio of positives over number of renal cells $(\mathrm{R})$ and ratio of positive cells over number of lymphocytes plus monocytes-macrophages (LM) were 35, 0.14 and 0.29, respectively.

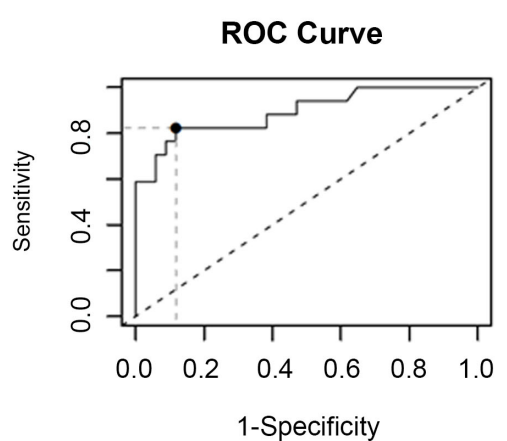

ROC Curve

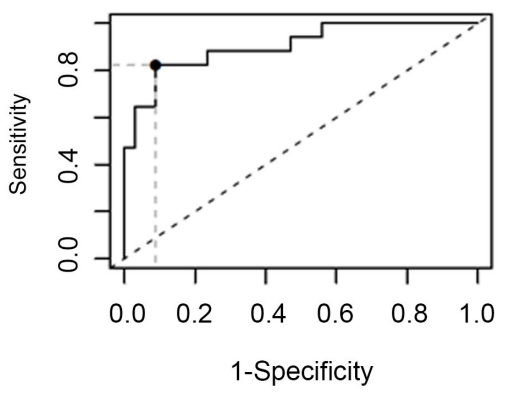

Distribution of Absolute.Value

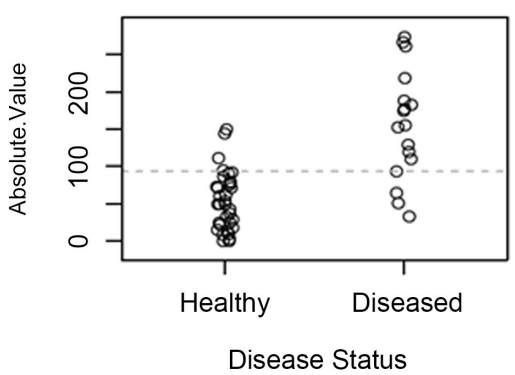

Distribution of Pos.Cel.R.Cel

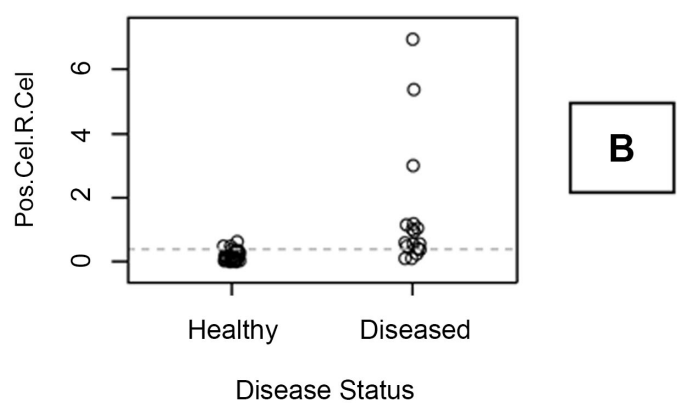


ROC Curve

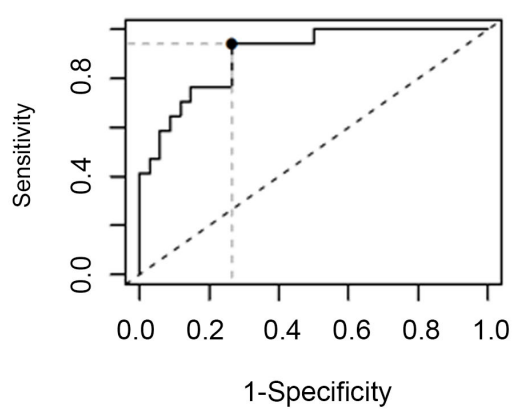

Distribution of Pos.Cel.LyMo

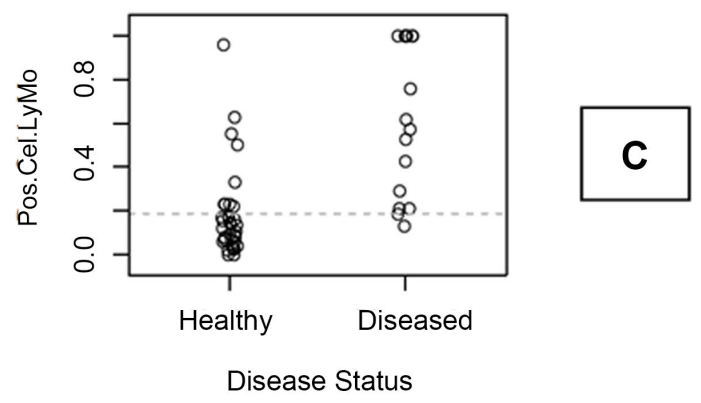

Figure 1. IRF3 regulatory factor could segregate Non-Rejection (NR) patients from those with Acute Rejectin (AR). The score model was practiced on $34 \mathrm{NR}$ and $17 \mathrm{AR}$ samples to generate a scale Q score ranging from 0 to 300 for Absolute Value (AV), from 0 to 7 and 0 to 1 for Pos.Cel.R. and Pos.Cel.LyMo ratios respectively. The AUC of the ROC curves and the disribution of NR and AR are shown in the figure. (A) For AV the Youden optimal cut-off method set a threshold at 93 with a corresponding sensitivity of $82.4 \%$ and specificity of $88.2 \%$. The AUC of the ROC curve was 0.894 ( $\mathrm{P}<0.0001)$; (B) For the ratio PosCelR the Youden optimal cut-off method set a threshold at 0.382 with a corresponding sensitivity of $82.4 \%$ and specificity of $91.2 \%$. The AUC of the ROC curve was $0.904(\mathrm{P}<0.0001)$; (C) For the ratio PosLM the Youden optimal cut-off method set a threshold at 0.187 with a corresponding sensitivity of $94.1 \%$ and specificity of 73.5 The AUC of the ROC curve was $0.894(\mathrm{P}<0.0001)$.

In Table 6 we present the results for IFN $\alpha$ in supernatants of Fnab cultures and in Table 7 the results for acute rejection diagnostic performance which were surprisingly very high by using the cut-off $104 \mathrm{pg} / \mathrm{ml}$. We did not find any significant correlation between IFN $\alpha$ synthesis by Fnab cultures and drug blood levels or serum creatinine. In Figure 3 are the IFN $\alpha$ results assessed for AUC of the ROC curves and distribution of non-rejection patients and acute rejection group.

\section{Discussion}

Our study done in a group of human renal transplant cases highlights a clear significant difference in IRF3 and IRF7 expression on graft-infiltrating cells obtained from Fnab samples of acute rejection patients. Furthermore, those cells when incubated under appropriate conditions, synthesize large amounts of IFNa as compared to stable ones. There have been few reports on these factors among KTx [21] and as far as we are aware, ours is the first report on diagnostic ability of these factors in KTx or in any organ transplant for that matter.

The low number of cases did not allow a subdivision of different kinds of rejection, especially the cellular and humoral types, and of irreversible rejection crisis (we were able to include only two such episodes), although a non-significant positive correlation was observed between serum creatinine and IRF3 expression within the acute rejection group suggesting the higher expression of IRF3 on graftinfiltrating cells the higher the rejection severity grade. Also, we noticed a nonsignificant IRF3 down-regulation among IL-2R $\alpha$-chain antibody-treated KTx, a therapy which we have previously reported to significantly down-regulate TLR2 and 
ROC Curve

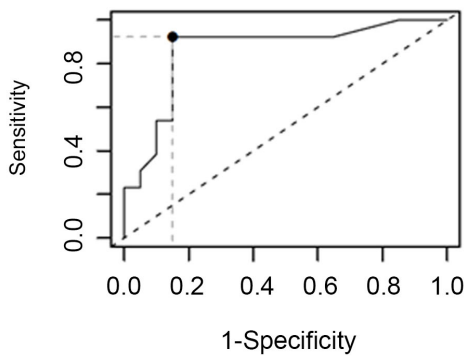

ROC Curve

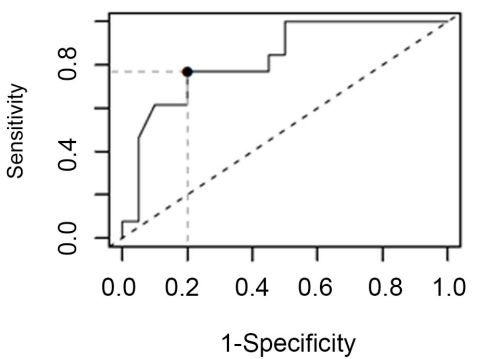

ROC Curve

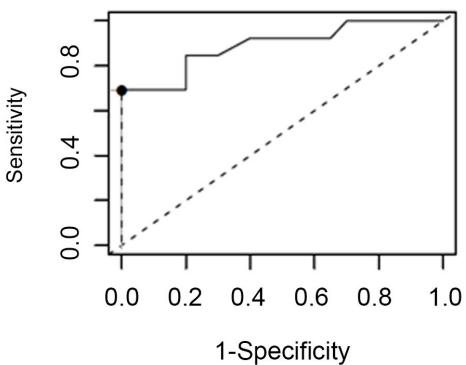

Distribution of Absolute.Value

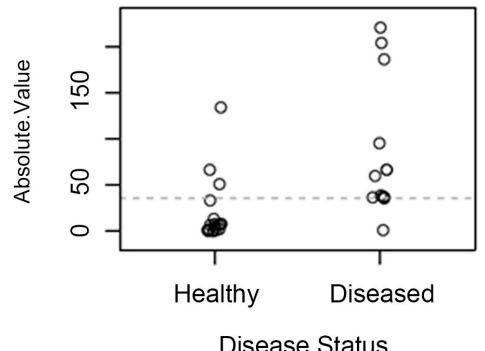

Distribution of Pos.Cel.R.Cel

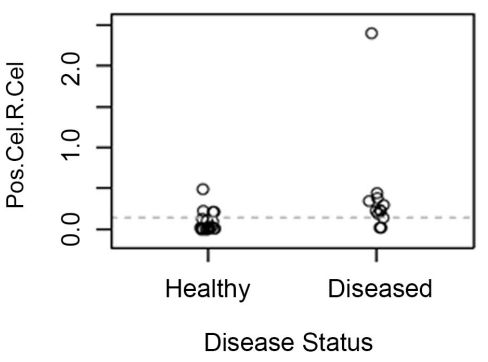

Distribution of Pos.Cel.LyMo

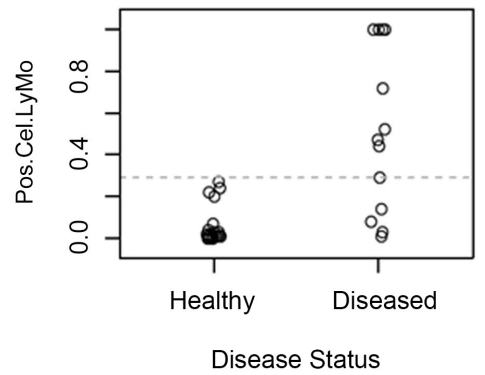

A

Figure 2. IRF7 regulatory factor could segregate Non-Rejection (NR) patients from those with Acute Rejectin (AR). The score model was practiced on $20 \mathrm{NR}$ and $13 \mathrm{AR}$ samples to generate a scale Q score ranging from 0 to 200 for Absolute Value (AV), from 0 to 2.5 and 0 to 1 for PosCelR and PosLM ratios respectively. The AUC of the ROC curves and the disribution of NR and AR are shown in the figure. (A) For AV the Youden optimal cut-off method set a threshold at 35 with a corresponding sensitivity of $92.3 \%$ and specificity of 85.0\%. The AUC of the ROC curve was 0.860 ( $\mathrm{P}<0.0001$ ); (B) For the ratio PosCelR the Youden optimal cut-off method set a threshold at 0.14 with a corresponding sensitivity of $76.9 \%$ and specificity of $80.0 \%$. The AUC of the ROC curve was 0.827 ( $\mathrm{P}<0.0001)$; (C) For the ratio PosLM the Youden optimal cut-off method set a threshold at 0.29 with a corresponding sensitivity of $69.2 \%$ and specificity of $100 \%$. The AUC of the ROC curve was 0.890 $(\mathrm{P}<0.0001)$.

Table 6. IFN $\alpha$ results in Fnab culture supernatants.

\begin{tabular}{cccc}
\hline A1 & A2 & B & Mann-Whitney \\
$\mathrm{n}=34$ & $\mathrm{n}=14$ & $\mathrm{n}=15$ & $\mathrm{P}$ \\
\hline $75.1 \pm 14.4$ & $63.1 \pm 14.1$ & $212 \pm 216$ & A1 vs $A 2: 0.20$ \\
{$[62-87]$} & {$[52-72]$} & {$[105-178]$} & A vs B: 0.0001 \\
\hline
\end{tabular}

Group A1: stable cases; A2: Fnab on days 7 in cases treated with Rapamycin; group B: acute rejection group. Results expressed as $\mathrm{pg} / \mathrm{ml}$; values given as median $\pm \mathrm{SD}$ and lower and upper quartiles between brackets. 
Table 7. IFN $\alpha$ diagnostic performance for a cut-off point of 104.

\begin{tabular}{cc}
\hline IFNa & value \\
\hline Sensitivity & 0.867 \\
Specificity & 1.000 \\
Positive predictive value & 1.000 \\
Negative predictive value & 0.960 \\
Positive likelihood ratio & $\infty$ \\
Negative likelihood ratio & 0.133 \\
\hline
\end{tabular}
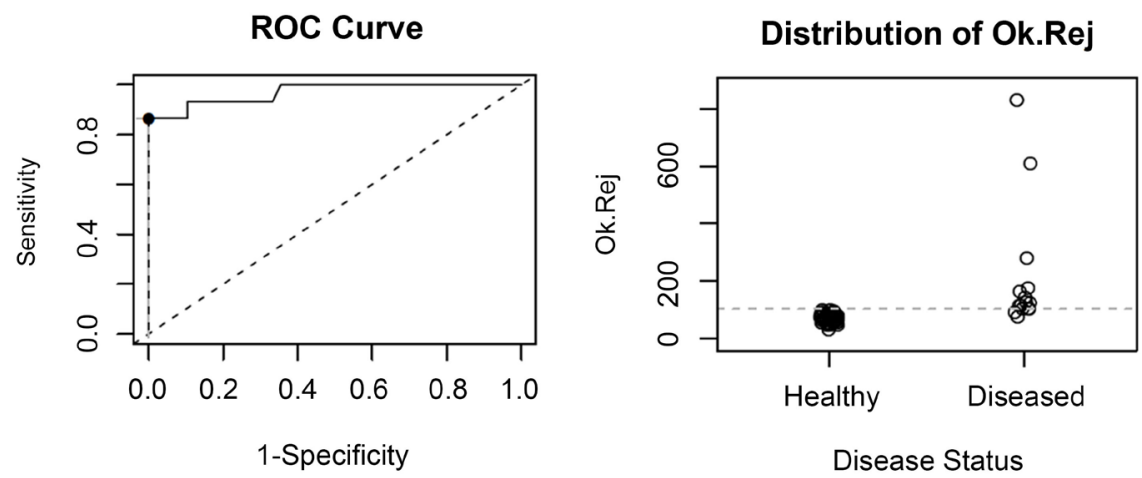

Figure 3. The synthesis of IFNa growth factor could discriminate Non-Rejection (NR) patients from those with Acute Rejectin (AR). The score model was practiced on $48 \mathrm{NR}$ and $15 \mathrm{AR}$ samples to generate a scale Q score ranging from 33 to $831 \mathrm{ng} / \mathrm{mL}$. The AUC of the ROC curves and the disribution of NR and AR are shown in the figure. The Youden optimal cut-off method set a threshold at 104 with a corresponding sensitivity of $86.7 \%$ and specificity of $100 \%$. The AUC of the ROC curve was $0.970(\mathrm{P}<0.0001)$.

TLR9 expression [27], but IRF7 displayed an opposite behaviour under this therapy. On the other hand, quadruple therapy is associated with a non-significant down-regulation of IRF7 as compared to triple therapy and we regret that we could not make the same comparison for IRF3 because of the scarcity of samples. Moreover, we did not observe a significant correlation between IFN $\alpha$ quantity measured in the Fnab culture supernatants with serum creatinine or with blood drug levels.

We were surprised by the very high diagnostic performance of IRF3, IRF7 and IFN $\alpha$, each of them displaying a positive predictive value higher than 0.8 and a negative predictive value higher than 0.90 . Such as we were anticipating that some of the high values could be confounded by the events pertaining to the IRI process, nonetheless that turned not to be the case, as can be also inferred by consulting the tables showing a clear separation between the upper quartiles in stable cases as compared to the lower quartiles in acute rejection ones. The most highlighting is brought by comparing the ratios of positive cells over the total of renal cells present where the lower quartile for acute rejection is more than the double of the upper quartile for stable cases for IRF3, and 40\% higher for IRF7 doing the same comparison. We remember that the renal function at the end of the first 
week among stable cases was still not good enough, the serum creatinine was 3.8 $\mathrm{mg} / \mathrm{dl}$, a value usually observed among KTx receiving organs from brain dead donors.

As depicted in Figure 4, IRF3 and IRF7 have been described as the principal mediators of IFN $\alpha$ [13]. While IRF3 is ubiquitously expressed, IRF7 is expressed at very low levels, except in plasmacytoid dendritic cells where it is relatively abundant [14]. Moreover, IRF7 expression is induced by IFN $\alpha$ resulting in a feedforward loop [31]. While several promoters of IRF3/IRF7 expression and activity could be advanced in the KTx context, TLR2, TLR4 and TLR9 will be among them [5]. TLR4 activates TRIF which via IKK kinase signalling will result in IRF3 activation [32]. Among several TLR4 ligands the DNA-binding protein high-mobility group box 1 and several cellular heat shock proteins will be relevant in IRI context [5]. Other TLR could be triggered during IRI. In a model of

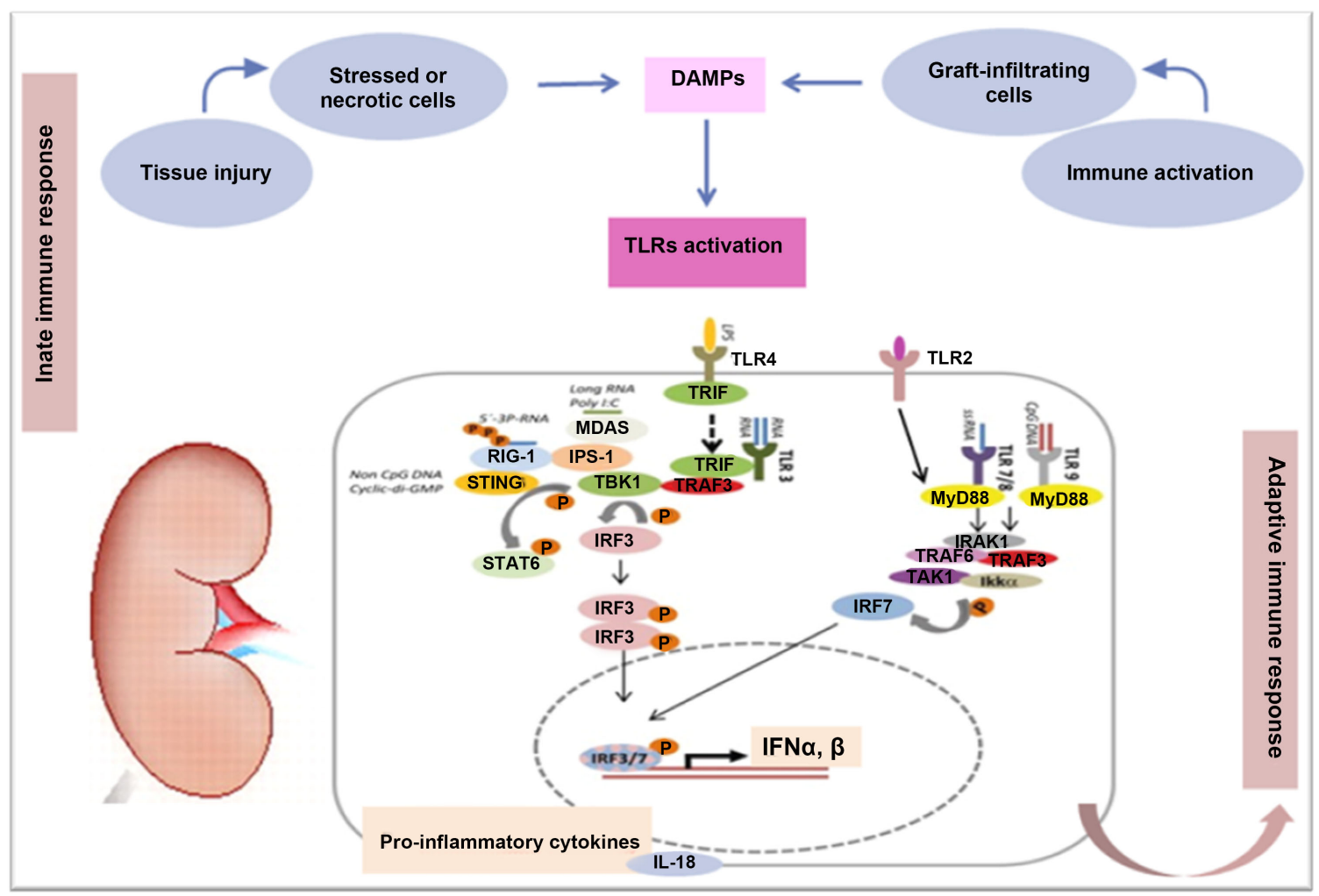

Figure 4. Schematic representation of IRFs and TLRs pathways in the context of kidney transplantation. The stimulation and engagement of several TLRs and other PRRs in immune cells launch signal and activate several effector mediators that are essential for TLR function and innate immune response. Downstream signalling inducing type I IFN, is mediated by initial binding to either MyD88 (TLR7/8/9) or TRIF (TLR3/4), followed by recruitment of multicomponent protein complexes. MyD88 recruited to TLR7/8/9 complexes with IRAK1, TRAF6, TRAF3, and the kinases TAK1 and IKKa, which phosphorylate and thus activate IRF7 to drive type I IFN expression. Also, in an independent MyD88 signalling, TRIF may be activated via IKKe and TBK1 phosphorylating IRF3 and IRF7. Secreted IFNs then bind and activate the type I IFN receptors in an autocrine or paracrine manner leading to enhance inflammation, proliferation and immune regulation. As a result, the adaptive immune response may enhance acute inflammatory responses controlled by innate immunity and so, in organ transplants graft function and survival may be influenced. TLRs, toll-like receptors; DAMPs, damage-associated molecular patterns; PRRs, pattern recognition receptors; IFN, interferon; IL, interleukin. This image was adapted from [43]. 
myocardial ischemia TLR2 and TLR9 mRNA expression was highly increased in the infarcted area together with IRF3 and IRF7 [33]. Something remote from our present study, IRF3/7 have been associated with systemic lupus [17] [34] and together with other IRFs they play several roles in cardiometabolic homeostasis [35]. Anyway, the message carried by our findings about IRF3 and IRF7 was greatly empowered by our data about IFN $\alpha$. The attention given to this interferon has been much less than that attributed to IFN $\gamma$ which is produced as a response to foreign antigens by $\mathrm{T}$ lymphocytes and natural killer cells both strongly associated with rejection. We have previously analysed IFN $\gamma$ synthesis by Fnab samples incubation and only found it in two of twenty-two stable KTx and five of twenty-two acutely rejecting KTx [36]. Certainly, the timing for IFN $\gamma$ measurement was not optimal as pointed by Danzer who showed that the peak of IFN $\gamma$ synthesis occurs by day six of incubation of mixed lymphocyte cultures [37]. We collected the culture supernatants at 48 hours because it was a good compromise to measure other growth factors we were also interested. However, in the same study [36] we did observe a significantly higher increase of IL-2 synthesis in acute rejection KTx, a difference reaching a good discriminatory power from stable cases. We searched for IL-12 that has been attributed a primordial role to the Thelper 1 subtype priming and subsequent IL-2 production [38]. Contrary to our anticipation, IL-12 was not increased among acute rejection cases [28], confirming other observations of lack of correlation between IL-12 mRNA in graft-infiltrating cells with acute rejection [39], but we did observe a significantly higher production of IL-18 among acute rejection KTx [29]. Although by itself IL-18 is able to stimulate T cells, taking good notice of the present results we surmise that the combination of IL-18 with IFN $\alpha$ will be very able to efficiently prime the adaptive immune response and specifically, of IL-2-producing $\mathrm{T}$ cells, among other consequences. This is not to dismiss the potential of IFN $\alpha$ by itself to strongly influence the events post-KTx. IFN $\alpha$ was able to induce KTx rejection when administered as therapy for hepatitis $\mathrm{C}$ patients submitted to transplantation between nine and 66 months before [25] and the same was even observed with combined liver-kidney recipients a combination associated with easier operational immune tolerance. Furthermore, liver transplant patients have presented different complications following IFN $\alpha$ treatment post-transplant, including acute rejection of previously immunologically stable grafts [26]. However, these situations, where these detrimental effects are observed with variable frequency but are far away from the one we are studying, the IRI period, when a diversity of stimuli turn around to prime the IFN $\alpha$ response [5] [40]. Also, the remarkable positive predictive value for acute rejection exhibited by IFN $\alpha$ is an indirect sign of its non-redundancy.

Our study suffers from a few limitations. It is a retrospective study where the number of samples is not large, which prevented the subdivision we were looking for regarding different types of acute rejection and different immunosuppressive drug protocols. So, we were restricted to study KTx treated with stan- 
dard of care immunosuppressive drugs. Furthermore, as a result of the limited amount of each sample we were unable to analyse both IRFs in the same patient sample. More relevant, following the restrictions inherent to human studies a sequential sampling of the same KTx could not be performed. Also, the timing for Fnab sampling among stable cases were concentrated at day seven post-transplant, with around $20 \%$ of Fnab done between day 7 and day 30 post-surgery, whilst for acute rejection patients Fnab sampling was guided by acute rejection appearance, which encompassed a wide period of time, at a median of $13.5 \pm 415$ days post-KTx, yet close to $60 \%$ of the events were observed during the first month. Anyway, the other later cases could only be better relieved from the IRI process making our data rather stronger than weaker.

It could also be said that our Fnab samples are contaminated by a variable amount of peripheral blood, which is true. However, we have repeatedly showed that the cell types present in Fnab are different from those present in the peripheral blood, implying that what we are seeing reflects, although with imperfections, the changes taking place inside the renal grafts that do not appear at the peripheral blood level [41] [42].

This acknowledged, we believe our data are rather consistent and produce further information on anti-allograft response, particularly during the important early period post-transplantation bringing to the forefront type I interferon and its main stimulators IRF3 and IRF7.

\section{Acknowledgements}

Dias-Pinto, P.X. and Oliveira, J.G.G. received a research fund from Novartis ${ }^{\circledR}$.

\section{Conflicts of Interest}

The authors declare no conflicts of interest regarding the publication of this paper.

\section{References}

[1] World Health Organisation (2015) Disease Burden and Mortality Estimates. WTO, Geneva.

https://www.who.int/healthinfo/global burden disease/estimates/en/index1.html

[2] Hart, A., Smith, J.M., Skeans, M.A., et al. (2019) OPTN/SRTR 2017 Annual Data Report: Kidney. American Journal of Transplantation, 19, 19-123.

[3] Rao, N.N. and Coates, P.T. (2018) Cardiovascular Disease after Kidney Transplant. Seminars in Nephrology, 38, 291-297. https://doi.org/10.1016/j.semnephrol.2018.02.008

[4] Salvadori, M., Rosso, G. and Bertoni, E. (2015) Update on Ischemia-Reperfusion Injury in Kidney Transplantation: Pathogenesis and Treatment. World Journal of Transplantation, 5, 52-67. https://doi.org/10.5500/wjt.v5.i2.52

[5] Nieuwenhuijs-Moeke, G.J., Pischke, S.E., Berger, S.P., Sanders, J.S.F., Pol, R.A., Struys, M.M.R.F., Ploeg, R.J. and Leuvenink, H.G.D. (2020) Ischemia and Reperfusion Injury in Kidney Transplantation: Relevant Mechanisms in Injury and Repair. Journal 
of Clinical Medicine, 9, 253.

[6] Erpiocum, P., Detry, O., Weekers, L., Bonvoisin, C., Lechanteur, C. and Briquet, A. (2014) Mesenchymal Stromal Cell Therapy in Conditions of Renal Ischemia/Reperfusion. Nephrology Dialysis Transplantation, 29, 1487-1493.

https://doi.org/10.1093/ndt/gft538

[7] Denecke, C. and Tullius, S.G. (2014) Innate and Adaptive Immune Responses Subsequent to Ischemia-Reperfusion Injury in the Kidney. Progrès en Urologie, 24, S13-S19.

[8] O’Neill, L.A. and Bowie, A.G. (2007) The Family of Five: TIR-Domain-Containing Adaptors in Toll-Like Receptor Signalling. Nature Reviews Immunology, 7, 353-364. https://doi.org/10.1038/nri2079

[9] Assadiasl, S., Mousavi, M.J. and Amirzagar, A. (2018) Toll-Like Receptor 4 in Renal Transplant. Experimental and Clinical Transplantation, 16, 245-252.

[10] Rusai, K., Sollinger, D., Baumann, M., et al. (2010) Toll-Like Receptors 2 and 4 in Renal Ischemia/Reperfusion Injury. Pediatric Nephrology, 25, 853-860.

[11] Bergler, T., Hoffmann, U., Bergler, E., Jung, B., Banas, M.C., Reinhold, S.W., Krämer, B.K. and Banas, B. (2012) Toll-Like Receptor 4 in Experimental Kidney Transplantation: Early Mediator of Endogenous Danger Signals. Nephron Experimental Nephrology, 121, e59-e70.

[12] Wu, H., Chen, G., Wyburn, K.R., et al. (2007) TLR4 Activation Mediates Kidney Ische$\mathrm{mia} /$ Reperfusion Injury. Journal of Clinical Investigation, 117, 2847-2859.

https://doi.org/10.1172/JCI31008

[13] Jefferies, C.A. (2019) Regulating IRFs in IFN Driven Disease. Frontiers in Immunology, 10, Article No. 325. https://doi.org/10.3389/fimmu.2019.00325

[14] Au, W.C., Moore, P.A., LaFleur, D.W., Tombal, B. and Pitha, P.M. (1998) Characterization of the Interferon Regulatory Factor-7 and Its Potential Role in the Transcription Activation of Interferon A Genes. Journal of Biological Chemistry, 273, 29210-29217. https://doi.org/10.1074/jbc.273.44.29210

[15] Brownell, J., Bruckner, J., Wagoner, J., et al. (2014) Direct, Interferon-Independent Activation of the CXCL10 Promoter by NF-kappaB and Interferon Regulatory Factor 3 during Hepatitis C Virus Infection. Journal of Virology, 88, 1582-1590. https://doi.org/10.1128/JVI.02007-13

[16] Xu, P., Bailey-Bucktrout, S., Xi, Y., et al. (2014) Innate Antiviral Host Defense Attenuates TGF-Beta Function through IRF3-Mediated Suppression of SMAD Signalling. Molecular Cell, 56, 723-737. https://doi.org/10.1016/j.molcel.2014.11.027

[17] Honda, K., Yanai, H., Negishi, H., et al. (2005) IRF7 Is the Master Regulator of Type-I Interferon-Dependent Immune Responses. Nature, 434, 772-777. https://doi.org/10.1038/nature03464

[18] Chattopadhyay, S., Veleeparambil, M., Poddar, D., et al. (2015) EGFR Kinase Activity Is Required for TLR4 Signalling and the Septic Shock Response. EMBO Reports, 16, 1535-1547. https://doi.org/10.15252/embr.201540337

[19] Zhao, G.-N., Jiang, D.-S. and Li, H. (2015) Interferon Regulatory Factors: At the Crossroads of Immunity, Metabolism, and Disease. Biochimica et Biophysica Acta, 1852, 365-378.

[20] Holm, C.K., Paludan, S.R. and Fitzgerald, K.A. (2013) DNA Recognition in Immunity and Disease. Current Opinion in Immunology, 25, 13-18.

https://doi.org/10.1016/j.coi.2012.12.006

[21] Morgun, A., Shulzhenko, N., Perez-Diez, A., Diniz, V.Z., Sanson, G.F., Almeida, D.R., Matzinger, P. and Gerbase-De-Lima, M. (2006) Molecular Profiling Improves Di- 
agnoses of Rejection and Infection in Transplanted Organs. Circulation Research, 98, E74-E83.

[22] Nasiri, M., Geramizadeh, B., Nabavizadeh, S.H., Male-Hosseini, S.A., Karimi, M.H. and Saadat, I. (2018) mRNA Expression of Interferon Regulatory Factors during Acute Rejection of Liver Transplants in Patients with Autoimmune Hepatitis. International Journal of Organ Transplantation Medicine, 9, 34-40.

[23] Li, S., Gong, M., Zhao, F., Shao, J., Xie, Y., Zhang, Y. and Chang, H. (2018) Type I Interferons: Distinct Biological Activities and Current Applications for Viral Infection. Cellular Physiology and Biochemistry, 51, 2377-2396. https://doi.org/10.1159/000495897

[24] Ambrus, J.L., Chadha, K.C., Anthone, S., Athone, R. and Sayyid, S. (1993) The AlphaInterferon System in Patients Undergoing Renal Transplantation and Treated with Cyclosporine and Prednisolone. Surgery, Gynecology and Obstetrics, 176, 588-590.

[25] Magnone, M., Holley, J.L., Shapiro, R., et al. (1995) Interferon- $\alpha$-Induced Acute Renal Allograft Rejection. Transplantation, 59, 1068-1070. https://doi.org/10.1097/00007890-199504150-00030

[26] Selzner, N., Guindi, M., Renner, E.L. and Berenguer, M. (2011) Immune-Mediated Complications of the Graft in Interferon-Treated Hepatitis C Positive Liver Transplant Recipients. Journal of Hepatology, 55, 207-217. https://doi.org/10.1016/j.jhep.2010.11.012

[27] Xavier, P.D.P., Alves, H., Oliveira, J.G.G., Santos-Araújo, C.A.R., Roncon-Albuquerque, R.L.F., Leite-Moreira, A.L.F., Ribeiro, A.S.L. and Sampaio-Norton, S.M.M. (2009) Direct Downregulation of Toll-Like Receptors by Anti-IL-2 Alpha Chain-Receptor Antibody in Cadaver Kidney Transplant Recipients. Transplantation, 88, 848-849 https://doi.org/10.1097/TP.0b013e3181b4e727

[28] de Oliveira, J.G.G., Xavier, P., Ramos, J., Sampaio, S., Magalhaes, M., Mendes, A. and Pestana, M. (2002) Cultures of Kidney Transplant Fine-Needle Aspiration Samples from Rejection-Free Patients Produce a Specific Antidonor Response Suppressive Factor. Nephron, 91, 637-645. https://doi.org/10.1159/000065025

[29] de Oliveira, J.G.G., Xavier, P.D.P., Sampaio, S.M., Tavares, S.I. and Mendes, A.A. (2002) The Synthesis by Fine-Needle Aspiration Biopsy Cultures of IL-7, IL-16, and IL-18 Is Significantly Associated with Acute Rejection in Kidney Transplants. Nephron, 92, 622-628. https://doi.org/10.1159/000064106

[30] Haÿry, P. (1989) Fine-Needle Aspiration Biopsy in Renal Transplantation. Kidney International, 36, 130-141. https://doi.org/10.1038/ki.1989.172

[31] Marie, I., Durbin, J.E. and Levy, D.E. (1998) Differential Viral Induction of Distinct Interferon-Alpha Genes by Positive Feedback through Interferon Regulatory Factor-7. EMBO Journal, 17, 6660-6669. https://doi.org/10.1093/emboj/17.22.6660

[32] Fitzgeral, K.A., McWhirter, S.M., Faia, K.L., et al. (2003) IKKe and TBK1 Are Essential Components of the IRF3 Signaling Pathway. Nature Immunology, 4, 491-496. https://doi.org/10.1038/ni921

[33] Becher, P.M., Hinrichs, S., Fluschnik, N., et al. (2018) Role of Toll-Like Receptors and Interferon Regulatory Factors in Different Experimental Heart Failure Models of Diverse Etiology: IRF7 as Novel Cardiovascular Stress-Inducible Factor. PLoS $O N E, 13, \mathrm{e} 0193844$.

[34] Mai, L., Asaduzzaman, A., Noamani, B., Fortin, P.R., Gladman, D.D., Touma, Z., Urowitz, M.B. and Wither, J. (2021) The Baseline Interferon Signature Predicts Disease Severity over the Subsequent 5 Years in Systemic Lupus Erythematosus. Arthritis Research \& Therapy, 23, 29.

[35] Zhang, Y. and Li, H. (2017) Reprogramming Interferon Regulatory Factor Signal- 
ling in Cardiometabolic Diseases. Physiology, 32, 210-223. https://doi.org/10.1152/physiol.00038.2016

[36] Oliveira, G., Xavier, P., Murphy, B., Neto, S., Mendes, A., Sayegh, M.H. and Guerra, L.E. (1998) Cytokine Analysis of Human Renal Allograft Aspiration Biopsy Cultures Supernatants Predicts Acute Rejection. Nephrology Dialysis Transplantation, 13, 417-422. https://doi.org/10.1093/oxfordjournals.ndt.a027839

[37] Danzer, S.G., Kirchner, H. and Rink, L. (1994) Cytokine Interaction in Human Mixed Lymphocyte Culture. Transplantation, 57, 1638-1642. https://doi.org/10.1097/00007890-199457110-00017

[38] Nickerson, P., Steurer, W., Steiger, J., Zheng, X., Steele, A.W. and Strom, T.B. (1994) Cytokines and the Th1/Th2 Paradigm in Transplantation. Current Opinion in Immunology, 6, 757-764. https://doi.org/10.1016/0952-7915(94)90081-7

[39] Zuo, X.-J., Jordan, S.C., Wilkinson, A., Danovitch, G.M., Barba, L., Scwieger, J. and Nast, C.C. (1995) Interleukin-12 mRNA Levels in Renal Allograft Fine-Needle Aspirates Do Not Correlate with Acute Transplant Rejection. Transplantation, 60, 1360-1362.

[40] Anders, H.-J., Lichtnekert, J. and Allam, R. (2010) Interferon- $\alpha$ and - $\beta$ in Kidney Inflammation. Kidney International, 77, 848-854. https://doi.org/10.1038/ki.2010.71

[41] Oliveira, J.G.G., Ramos, J.P., Xavier, P., Magalhães, M., Mendes, A. and Guerra, L.E.R. (1997) Analysis of Fine-Needle Aspiration Biopsies by Flow Cytometry in Kidney Transplant Patients. Transplantation, 64, 97-102. https://doi.org/10.1097/00007890-199707150-00018

[42] Oliveira, J.G.G., Ramos, J.P., Xavier, P., Sampaio, S., Magalhães, M., Mendes, A.A. and Pestana, M. (2001) Microemulsion Cyclosporine Formulation, in Contrast to the Old Formulation, Widens the T Lymphocyte Subsets Differences between Stable and Acute Rejection of Kidney Transplants. Nephrology Dialysis Transplantation, 16, 1256-1261. https://doi.org/10.1093/ndt/16.6.1256

[43] Prinz, M. and Knobeloch, K.-P. (2012) Type I Interferons as Ambiguous Modulators of Chronic Inflammation in the Central Nervous System. Frontiers in Immunology, 3, 67. https://doi.org/10.3389/fimmu.2012.00067

\section{Abbreviations}

Fine-needle aspiration biopsies: Fnab

IFN: interferon

IRF: interferon regulatory factors

Kidney transplants: KTx 